\title{
Synchronization of the Cell Division Cycle of Chlamydomonas eugametos
}

\author{
By R. DEMETS, A. M. TOMSON, E. T. H. RAN, C. A. M. SIGON, \\ D. STEGWEE AND H. VAN DEN ENDE* \\ Plantenfysiologisch Laboratorium, Universiteit van Amsterdam, Kruislaan 318, \\ 1098 SM Amsterdam, Holland
}

(Received 2 April 1985; revised 8 July 1985)

\begin{abstract}
A description is given of the vegetative growth of Chlamydomonas eugametos in liquid cultures. When a circadian light/dark regime is applied, cell division takes place during the dark periods and the release of the daughter cells is phased round the dark/light transitions. However, as long as the cultures are agitated, only a fraction of the cells divides during any dark period. The mother cells stick firmly to the surface of the culture vessels, making it possible to separate them from the non-dividing cells. They release daughter cells that show cell cycles of $1 \times 24 \mathrm{~h}, 2 \times 24 \mathrm{~h}$ and longer. Only in static cultures do all cells divide synchronously every $24 \mathrm{~h}$. To explain these results, a correlation is postulated between the chance of settling and the length of the division cycle.
\end{abstract}

\section{INTRODUCTION}

Chlamydomonas is a biflagellated unicellular green alga, reproducing vegetatively by cell division. When grown in a liquid medium, the cells retract their flagella before division (Cavalier-Smith, 1974). The protoplast divides into a number of daughter cells which are released after enzymic dissolution of the mother cell wall. In $C$. eugametos, vegetative reproduction is terminated, when the culture medium runs out of nutrients, by a gametogenic cell division (Tomson et al., 1985). To take a closer look at the division cycle of $C$. eugametos, we tried to synchronize the cells by the method of Bernstein, which was successfully applied to the closely related species $C$. moewusii (Bernstein, 1960; Kates \& Jones, 1964), and is still frequently used to synchronize $C$. reinhardtii (Surzycki, 1971; Cavalier-Smith, 1974; Spudich \& Sager, 1980). This method uses a phasing of the cell cycle by imposing a circadian light/dark regime on liquid cell cultures. In early reports by Wetherell (1958) and Bernstein (1960) it was mentioned that light/dark shifts are not sufficient to synchronize $C$. eugametos. In this paper, we demonstrate that $C$. eugametos can be synchronized in this way provided that the cultures are neither shaken nor aerated.

\section{METHODS}

Chlamydomonas eugametos strain UTEX 10 (mating-type minus) from the Algal Collection at the University of Texas at Austin, USA, was used. Axenic, rotary shaken (40 r.p.m.) cultures in Fernbach flasks containing $1000 \mathrm{ml}$ liquid medium [minerals as used by Kates \& Jones (1964); trace elements according to Wiese (1965); and FeEDTA according to Jones (1962)], aerated by compressed air, were grown at $19^{\circ} \mathrm{C}$ on a $16 \mathrm{~h}$ light $/ 8 \mathrm{~h}$ dark regime. Cultures in Petri dishes containing $20 \mathrm{ml}$ liquid medium were grown in a similar way, but were not aerated. Illumination was provided by white fluorescent tubes $\left(3500 \mathrm{~lx} ; 4.2 \mathrm{~W} \mathrm{~m}^{-2}\right)$, unless indicated otherwise. Static cultures were neither aerated nor shaken. To determine cell densities, culture samples were fixed with glutaraldehyde (final concn $1.25 \%, \mathrm{v} / \mathrm{v}$ ) and counted in a haemocytometer. Cells were photographed with a Zeiss photomicroscope and Agfapan 25 film. 


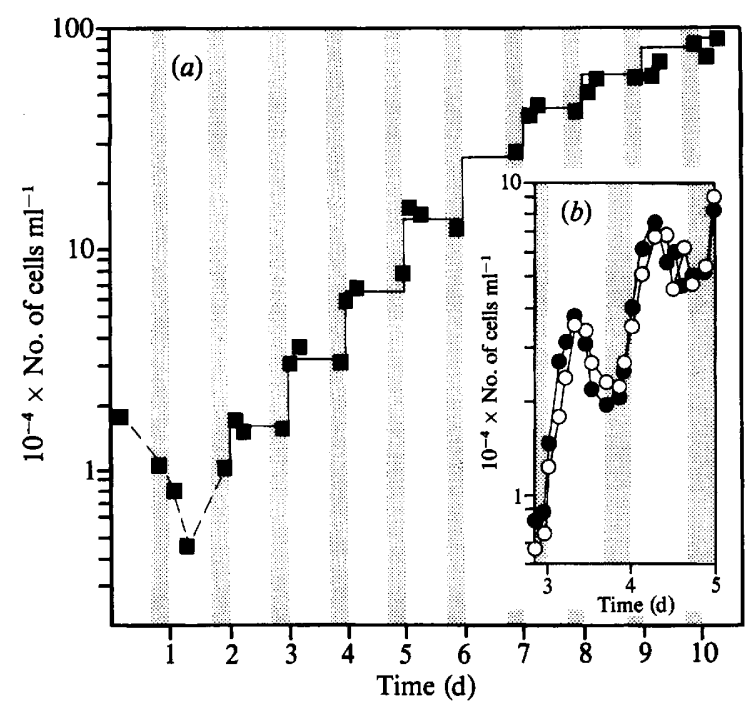

Fig. 1. (a) Division rhythm in an agitated culture of $C$. eugametos. (b) Settling rhythm in two agitated parallel cultures of $C$. eugametos. Shaded areas indicate dark periods.

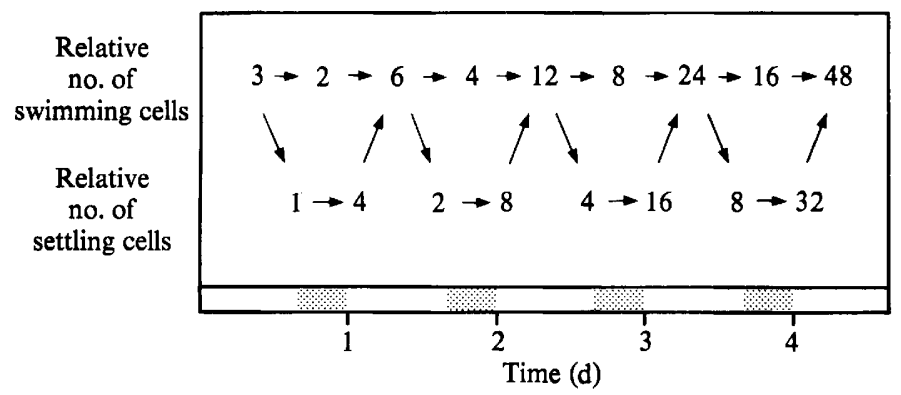

Fig. 2. Growth pattern model of $C$. eugametos explaining Fig. 1. The model accounts for the following observations: (1) a doubling of the cell number every $24 \mathrm{~h}$; (2) one out of three cells settles and divides during every $24 \mathrm{~h}$ period; (3) each dividing cell gives four daughter cells. Shaded areas indicate dark periods.

\section{RESULTS}

\section{Cell division and settling rhythms}

When $C$. eugametos was grown under a regime of $16 \mathrm{~h}$ light $/ 8 \mathrm{~h}$ dark, the cell number doubled daily during the exponential phase of growth (Fig. $1 a$ ). The cell density increased in daily bursts as daughter cells were released around the transition from dark to light. When cells were sampled at shorter intervals, a pattern of alternating peaks and dips was found (Fig. $1 b$ ). Every day the cell number dropped to a minimum in the dark period because cells disappeared from the suspension by sticking to the surface of the culture flasks. By placing a series of glass slides on the bottom of the flasks and removing them one by one over a $24 \mathrm{~h}$ period, cell-to-glass adhesion was microscopically confirmed. As expected, towards the end of the light period, the slides became increasingly occupied with cells. Towards the end of the dark period, these settling cells could be seen to divide predominantly into four daughter cells. At the dark/light transition, the cell division was completed when the daughter cells were released from the mother cell wall and left the slides. Furthermore, from the absence of dividing cells in samples taken from the cell suspension, at any time, it was concluded that only the settling cells were involved in cell division. The settling rhythm started in the lag phase and continued during the exponential and stationary phases. 


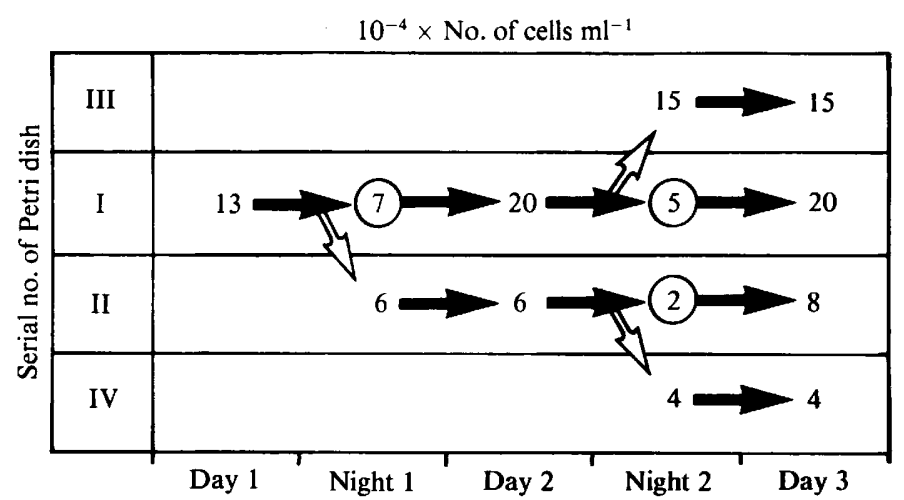

Fig. 3. Cell multiplication of $C$. eugametos grown in agitated Petri dishes. Roman numbers refer to individual Petri dishes. Other numbers refer to cell densities. Encircled numbers refer to the cells that became attached to the dish. Open arrows represent a transfer of the non-adherent cells to a new Petri dish. The results are mean values of duplicate determinations.

\section{Length of the cell division cycle}

The cell number doubled daily (Fig. 1), while each dividing cell generally produced four daughters, giving a mean generation time of $48 \mathrm{~h}$. By comparing the maxima and the minima of the undulating cell density during the growth phase, it was found that each night about one-third of the cell population was settling. When these data were combined in a model (Fig. 2), the growth pattern of Fig. 1 could be explained.

To determine the actual length of the division cycle, culture flasks were emptied in the middle of the dark period. By doing so, the non-dividing swimming cells were separated from the sticking dividing cells. The flasks were refilled with cell-free medium from a parallel culture. As expected, at the dark/light transition, all cells released their daughters. The growth of this population of synchronously born cells was followed for $2 \mathrm{~d}$. The cells multiplied during both the first and second day after their birth. So at least a fraction of the cells divided after $24 \mathrm{~h}$. Since the mean generation time was $48 \mathrm{~h}$, division times of $24 \mathrm{~h}, 48 \mathrm{~h}$ and, additionally, $72 \mathrm{~h}$ or even longer could occur. To test this, the multiplication of both the sticking and swimming cells was studied. After establishing that growth rate, percentage of settling cells and number of daughters per cell remained unaltered, cells were grown in rotary shaken Petri dishes, giving the advantage of easy microscopical observation of the cells. Fig. 3 shows the set-up and the results of these experiments. On the first day, Petri dish I was filled with $20 \mathrm{ml}$ cell suspension at $13 \times 10^{4}$ cells $\mathrm{ml}^{-1}$ from a Fernbach flask culture. During the first night, the cell density dropped to $6 \times 10^{4}$ cells $\mathrm{ml}^{-1}$. The suspension of non-dividing cells was poured out into Petri dish II, while the dividing cells remained in Petri dish I, their number equivalent to a cell density of $7 \times 10^{4}$ cells $\mathrm{ml}^{-1}$. Then, Petri dish I was refilled with cell-free medium. Transfers of cell suspensions on the second night, introducing Petri dishes III and IV, were done in a similar way. The experiment shows that 5 out of every 20 newly born cells on day 2 in Petri dish I divided during night 2, confirming the finding that at least a part (in this case $25 \%$ ) of the cells had a division time of $24 \mathrm{~h}$. Petri dish IV contained cells that had not divided during night 1 , nor during night 2 . So, on day 3 these cells were more than $48 \mathrm{~h}$ old and they were certainly viable because they were actively motile. This supports our assumption that cell cycle lengths of $72 \mathrm{~h}$ or even longer might occur, as well as the more common 48 and $24 \mathrm{~h}$ periods.

\section{Synchronization of the cell division cycle}

Numerous attempts were made to obtain synchrony by changing the culture conditions. Cells were cultivated in different media, with or without additional $\mathrm{CO}_{2}$, at different light intensities and different light/dark regimes. In all cases, only a fraction of the cells divided every $24 \mathrm{~h}$. Finally, we discovered that $C$. eugametos cells divided synchronously every $24 \mathrm{~h}$, provided that 

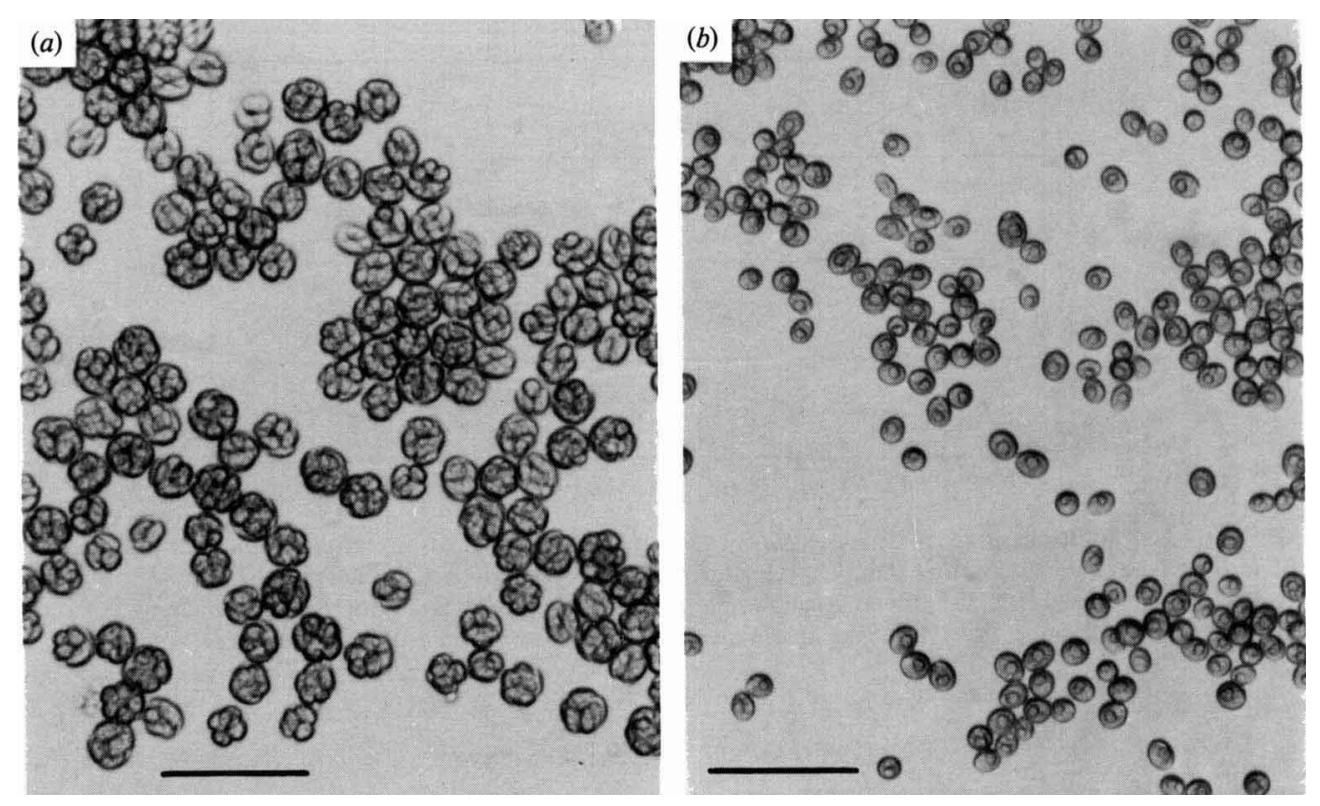

Fig. 4. Synchronous cell division in a static liquid culture of $C$. eugametos grown under light/dark (illumination $7000 \mathrm{~lx} ; 8.4 \mathrm{~W} \mathrm{~m}^{-2}$ ). (a) Dividing cells, floating in a monolayer on top of the liquid medium, $2 \mathrm{~h}$ before the dark/light transition. Note that at $7000 \mathrm{~lx}$ cells civide into four or eight daughters. (b) New-born cells just before they re-enter the cell suspension, $2 \mathrm{~h}$ after the dark/light transition. Bars, $50 \mu \mathrm{m}$.

Table 1. Growth of C. eugametos in static and shaken cultures

Cell densities are given that were reached after hatching. On day 1 a part of the cell suspension of a shaken Fernbach flask culture was poured out into static Petri dishes. The results are means of a representative experiment (carried out in duplicate) of a series of three.

$\begin{array}{ccr}\text { Day } & \overbrace{\text { Shaken }}^{10^{-4} \times \text { No. of cells } \mathrm{ml}^{-1}} \\ 1 & 14.5 & \text { Static } \\ 2 & 30 \cdot 3 & 14.5 \\ 3 & 45.9 & 69.5 \\ 4 & 78.8 & 230 \cdot 3 \\ & & 414.5\end{array}$

the culture vessels were not agitated or aerated (Fig. 4). In these static cultures, all cells left the suspension towards the end of the light period to form a monocellular layer on the surface of the culture medium. The floating cells excreted a thin film of mucilage that could be stained with periodic acid-Schiff reagent and, to a certain extent, with Coomassie Brilliant Blue, suggesting that polysaccharides and proteins were involved. Cells in the monolayer divided synchronously and released their daughter cells, as usual, at the dark/light transition. After hatching, the monolayer of cells and mucilage gradually dissolved and the culture returned to a suspension of swimming cells. Synchrony could be maintained for at least three rounds of cell division during the exponential growth phase of the culture. As higher cell densities were reached, the monolayer gradually transformed into a multilayer. The growth rate in the static cultures exceeded the growth rate in partially synchronous shaken cultures (Table 1).

\section{DISCUSSION}

Circadian cell division and settling rhythms have been described for $C$. reinhardtii by Straley \& Bruce (1979). They concluded that settling and cell division are independent. We found, 
similarly, that $C$. eugametos settled without cell division during the lag and the stationary phases of growth. In contrast, during the exponential phase settling and cell division went hand in hand. Kates \& Jones (1964) mentioned that settling cells of $C$. moewusii, unlike $C$. reinhardtii, adhered firmly to the bottom of the culture vessel. This behaviour was seen as a disadvantage, making it difficult to collect representative culture samples. However, the same phenomenon offers a simple method for splitting a culture into age classes by separating the dividing from the non-dividing cells. From the fact that the splitting did not significantly disturb the growth pattern, we conclude that the agitated cultures actually consisted of several classes of cells running $n \times 24$ h out-of-phase in respect of their division cycle. This means that there was a wide, discontinuous variability in both generation time and age among the individual cells, reminiscent of the dinoflagellates Gonyaulax polyedra and Gymnodinium splendens, as described by Sweeney (1969). She mentioned that for synchrony it would be necessary to find environmental conditions in which all cells can prepare for division in a single day. For C. eugametos we found that this meant keeping the cells totally undisturbed. Before cell division, cells stuck to the culture vessel in agitated cultures, or started floating passively on top of the culture medium in static cultures. In both cases, the cells left the suspension, settled down and retracted their flagella. The thin film excreted by the floating cells probably consisted of the same material that enabled the cells to stick to solid surfaces. Excretion of polysaccharides and proteins by Chlamydomonas has been described previously (Nalewajko, 1978).

The question arises as to what disturbs synchrony when cultures are agitated. Since we found that the growth rate in static cultures was higher than in shaken cultures, and that in shaken cultures the generation times were longer, we conclude that the agitation prevented the cells from entering the immobile phase and that they therefore postponed their division.

As to the control of the cell cycle, two models are in vogue: the deterministic and the probabilistic models (reviewed by Edmunds, 1982). Since we claim that the length of the cell cycle depends on the chance of settling, the cell cycle control of $C$. eugametos conforms to the probabilistic model. In the original form of the probability model (Smith \& Martin, 1973) the cell cycle is composed of an A-state and a B-phase. In the A-state the cell does not progress towards division but has a constant transition probability of entering the B-phase, which is made up of most of the conventional cell cycle and is completed in a constant period of time (Nurse, 1980). Most cell cycles might include probabilistic as well as deterministic components, and in fact the probability model of Smith \& Martin (1973) covers both, as the B-phase is completed in a constant time period and therefore is deterministic (Nurse, 1980). In our case, the A-state represents the swimming stage of the cells and the B-phase the immobile stage. Interestingly, the transition probability itself is obviously controlled by the agitation of the culture, i.e. purely by an environmental factor.

The static culture synchronizing system is an easy and simple method that adds a new category to the synchronization techniques that have appeared in the literature for Chlamydomonas (Lien \& Knutsen, 1979; Lloyd et al., 1982). And, finally, the problems of Wetherell (1958) and Bernstein (1960) concerning the synchronization of $C$. eugametos are resolved, making $C$. eugametos perhaps more similar to $C$. moewusii and $C$. reinhardtii in this field of work.

The authors thank Mr J. A. J. van der Meijden for his assistance with the photographic work and Dr J. L. Talen for helpful suggestions.

\section{REFERENCES}

BERNSTEIN, E. (1960). Synchronous division in Chlamydomonas moewusii. Science 131, 1528-1529.

CAVAlier-Smith, T. (1974). Basal body and flagellar development during the vegetative cell cycle and the sexual cycle of Chlamydomonas reinhardii. Journal of Cell Science 16, 529-556.

EDMUNDS, L. N., JR (1982). Circadian and infradian rhythms. In The Biology of Euglena. Vol. III. Physiology, pp. 53-139. Edited by D. E. Buetow. New York \& London: Academic Press.

JONES, R. F. (1962). Extracellular mucilage of the red alga Porphyridium cruentum. Journal of Comparative and Cellular Physiology 60, 61-64.

KATES, J. R. \& JONES, R. F. (1964). The control of 
gametic differentiation in liquid cultures of Chlamydomonas. Journal of Cellular and Comparative Physiology 63, 157-163.

LIEN, T. \& KNUTSEN, G. (1979). Synchronous growth of Chlamydomonas reinhardtii (Chlorophyceae): a review of optimal conditions. Journal of Phycology 15, 191-200.

Lloyd, D., Poole, R. K. \& Edwards, S. W. (1982). The Cell Division Cycle, pp. 64-65. New York \& London: Academic Press.

NALEWAJKo, C. (1978). Release of organic substances. In Handbook of Phycological Methods: Physiological and Biochemical Methods, pp. 389-398. Edited by J. A. Hellebust \& J. S. Craigie. Cambridge: Cambridge University Press.

NuRSE, P. (1980). Cell cycle control - both deterministic and probabilistic? Nature, London 286, 9-10.

SMith, J. A. \& Martin, L. (1973). Do cells cycle? Proceedings of the National Academy of Sciences of the United States of America 70, 1263-1267.

SPUDich, J. L. \& SAGER, R. (1980). Regulation of the Chlamydomonas cell cycle by light and dark. Journal of Cell Biology 85, 136-145.
Straley, S. C. \& Bruce, V. G. (1979). Stickiness to glass. Circadian changes in the cell surface of Chlamydomonas reinhardi. Plant Physiology 63, 1175-1181.

SURZYCKI, S. (1971). Synchronously grown cultures of Chlamydomonas reinhardi. Methods in Enzymology 23, 67-73.

SweEney, B. M. (1969). Rhythmic Phenomena in Plants, pp. 112-114. London \& New York: Academic Press. Tomson, A. M., Demets, R., Bakker, N. P. M., Stegwee, D. \& Ende, H. van DEN (1985). Gametogenesis in liquid cultures of Chlamydomonas eugametos. Journal of General Microbiology 131, 1553-1560.

WETHERELL, D. F. (1958). Obligate phototrophy in Chlamydomonas eugametos. Physiologia plantarum 11, 260-274.

WIESE, L. (1965). On sexual agglutination and matingtype substances (gamones) in isogamous heterothallic chlamydomonads. I. Evidence of the identity of the gamones with the surface components responsible for sexual flagellar contact. Journal of Phycology 1, 46-54. 